Rapid Reviews COVID-19

\title{
Review 3: "Seroprevalence of SARS-COV-2 Antibodies in Scottish Healthcare Workers"
}

\section{Petros Galanis ${ }^{1}$}

${ }^{1}$ National and Kapodistrian University of Athens: Ethniko kai Kapodistriako Panepistemio Athenon, Faculty of Nursing, Athens, Greece

Published on: Dec 17, 2020

DOI: $10.1162 / 2 \mathrm{e} 3983 f 5.1 \mathrm{~b} 18 \mathrm{~b} 0 \mathrm{c} 2$

License: Creative Commons Attribution 4.0 International License (CC-BY 4.0). 


\section{$\underline{\text { RR:C19 Evidence Scale rating by reviewer: }}$}

- Potentially informative. The main claims made are not strongly justified by the methods and data, but may yield some insight. The results and conclusions of the study may resemble those from the hypothetical ideal study, but there is substantial room for doubt. Decision-makers should consider this evidence only with a thorough understanding of its weaknesses, alongside other evidence and theory. Decisionmakers should not consider this actionable, unless the weaknesses are clearly understood and there is other theory and evidence to further support it.

*****************************************

\section{Review:}

Introduction should be expanded since there are a great number of studies and metaanalyses that estimate seroprevalence of SARS-COV-2 antibodies among healthcare workers, e.g. https://doi.org/10.1016/j.jhin.2020.11.008. Authors make comments about personal protective equipment and infection prevention and control in the Introduction section but these issues are not included in their study.

Methods section suffers from several flaws. This is definitely not a prospective observational study but a cross-sectional study with just one measurement for the participants. The response rate is not mentioned. Authors refer to two control groups in the Results section, but these groups are not clear in the Methods section. Also, random selection of blood samples taken at NHS Tayside General Practice Surgeries should be explained in detail. Statistical analysis is poor since the authors should perform multivariable logistic regression analysis with seroprevalence of SARS-COV-2 antibodies as the dependent variable to eliminate confounding. Afterwards, they should adjust their Discussion section according to the new findings.

The 95\% confidence interval for seroprevalence of SARS-COV-2 antibodies should be presented as well as adjusted seroprevalence for the sensitivity and the specificity of antibodies test.

Figures should be removed and tables with multivariable logistic regression analysis with seroprevalence of SARS-COV-2 antibodies as the dependent variable should be added. 
Recommendation: Manuscript needs major revision. Claims are not strongly supported, but may yield some insight by the data and methods used. Decision-makers should consider the claims in this study not actionable (except to prompt further research), unless the weaknesses are clearly understood and there is other theory and evidence to further support them based on the methods and data. 